\title{
An informational analysis of a visual search task
}

P. R. MEUDELL AND T. G. WHISTON
THE UNIVERSITY OF MANCHESTER

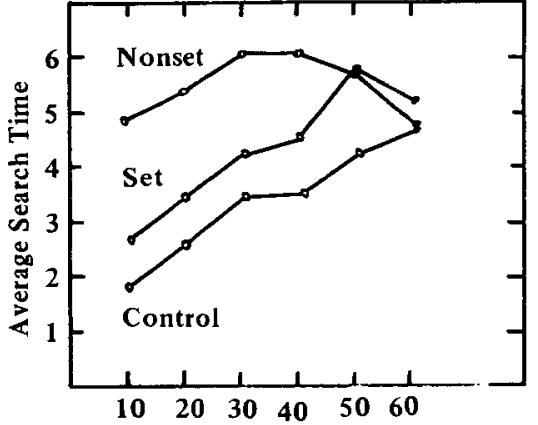

No. of Symbols with Target Color

Fig. 1. Average search time as a function of the number of symbols with the target's color for each condition (reproduced from Green \& Anderson, 1956).

search time). Green and Anderson plotted the average search time against the number of digits with the target's color. Their results are shown in Fig. 1.

Thus, the average search time for the set condition is primarily a function of the number of symbols of the target's color. When there is only one color (control condition), then the average search time is approximately a linear function of the total number of digits on display. Since the Ss did not know the color of the target in the nonset condition, the average search time should be a constant, independent of the number of symbols of the target's color. In fact, a horizontal line is not obtained; instead, they found an inverted-U relationship between average search time and the number of symbols of the target's color.

One can envisage this experiment as one in which the $E$ varies the amount of information in the stimulus matrix by increasing the total number of symbols in the control condition and by increasing the proportion of symbols with the target's color in the set condition.

Now, if we can quantify the amount of information for a given number of symbols in the control condition and a given proportion of target symbols in the set condition, then we should predict that if the two information measures are identical, the search times will also be identical. $I_{1}$ other words, the relationship betweelı search time and the amount of information will be the same, irrespective of set or control conditions. If we make the not unreasonable assumption that the Ss transmit information at a constant rate, one would expect a linear relation between search time and the total amount of information in the display.

Given this assumption, we specifically predict, therefore, that the information measure will reduce Green and Anderson's data to the same function relating search time to information on display, and, further, that the function will be linear.

The following analysis of Green and Anderson's first experiment assumes a systematic search strategy on the part of the $S$. We hypothesize, in fact, that the nonset group employed a redundant strategy; accordingly, we will apply the information measure only to the set and control conditions and postpone a discussion of the nonset condition until later in this paper.

For the control condition, there may be 10 symbols on display, each of which could be 1 of 60 possible numbers. Then, for this case, there are $10 \log _{2} 60$ bits of information. Similarly, with 20 numbers on display, there are $20 \log _{2} 60$ bits. The amount of information can be similarly calculated for all other values of $n$, where $n$ is the total number of symbols on display,

The crosses in Fig. 2 show these information values plotted as a function of search times. In the control condition, some of the cells in the matrix will be blank, and it could thus be argued that if the Ss process information about location, then the total information in any given display will be greater than $n \log _{2} 60$. For

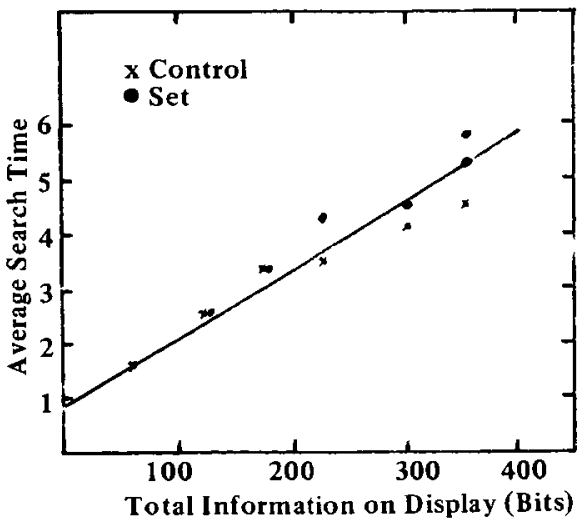

Fig. 2. Average search time as a function of total information on display. 
example, in the control condition containing 10 symbols and 50 blanks, the 10 symbols can occupy the 60 locations in $60 ! / 50 ! 10$ ! ways, and the symbols can be permuted in any of $60 ! / 50 !$ ways. Thus, on this analysis, the total information would be $\log _{2}[(60 ! / 50 ! 10 !)(60 ! / 50 !)]=94$ bits, not $10 \log _{2} 60=59$ bits. If, however, we assume that the Ss, in fact, scan the display sequentially, ignoring blanks (which are almost surely irrelevant to the Ss' search), and also assume that the $S$ s do not remember or cannot use the fact that certain previous numbers have been processed, then, for the $S$, the display does have $10 \log _{2} 60$ bits. We are explicitly assuming that the $S$ makes no use of the fact that with each successive symbol processed, the next symbol is more and more likely to be the target. In all subsequent calculations, we make these assumptions. The total information in any display is reduced by the strategy we suggest the $\mathbf{S}$ employs.

The information in the set condition (where the Ss know the color of the target) is calculated as follows: For the $S$, the first task is to identify the color of the number being searched. If there are 60 numbers, then each of these requires a one-bit decision (i.e., target color or not target color), a total of 60 bits in all. (This is so even though the proportion of target colors is not always equal to the proportion of nontarget colors, since the Ss were unaware on any trial of how many target-colored symbols would be presented. Incorporating these unequal proportions into the models slightly changes the slope of the function relating search time to total information.) Now, if the symbol is not of the target's color, no further examination of that symbol is required. If, however, the symbol is of the target's color, then a further examination with regard to target number is required. Since each symbol is 1 of 60 numbers, this examination requires $\log _{2} 60$ bits, and if there are, say, 10 symbols with the target's color, each requiring $\log _{2} 60$ bits, then the search for number among the symbols of the target's color requires 10 $\log _{2} 60$ bits. In total, therefore, there are $60+10 \log _{2} 60$ bits. Similarly, with 20 symbols of the target's color, there are $60+20 \log _{2} 60$ bits.

In general, if there are $m$ symbols with the target's color, the information in the display is given by $60+\mathrm{m} \log _{2} 60$. (Of course, when all the symbols are of the target color, then no search for color is required, and the information in the display in this case is simply $60 \log _{2} 60$.)

These information values are plotted as the solid points in Fig. 2, along with the control data, as a function of search time. It is clear that the data points from each of these two conditions lie on a straight line. This has been fitted by eye, the information-transmission rate being $80 \mathrm{bits} / \mathrm{sec}$. This value for the transmission rate, however, is that obtained assuming that the Ss searched the whole array. In fact, on the average, a $S$ will only have to search half of the array. Thus, the bits actually processed per unit time will be one-half of the value obtained from Fig. 2 (i.e., $40 \mathrm{bits} / \mathrm{sec}$ ).

\section{EXPERIMENT 2}

In Green and Anderson's second experiment, they again presented two-digit numbers in a matrix of 10 rows and six columns, and there were, again, three conditions: set, nonset, and control.

In this experiment, however, the number of possible target colors was increased Thus, in the set condition, there were two, three, or four colors, each consisting of 15 symbols. The $S$ was informed of the target color and had to scan 30 symbols for the two-color situation, 45 symbols for the three-color situation, and 60 symbols for the four-color situation.

The nonset condition was the same as for the set, except that the S was not told the target's color.

Finally, the control condition was the same as the control condition of the first experiment, except that the total numbers of symbols in this case were $15,30,45$, and 60.

Figure 3 shows Green and Anderson's results from this experiment.

Experiment 2 can be envisaged as subject to the same informational analysis as the former experiment. Thus, the data for the control condition in this experiment are calculated in the same way as for the control condition in the former experiment.

The set data consist of three data points. First, when there are 30 symbols, 15 of one color and 15 of the other, and since the Ss know the target color, there are 30 one-bit alternatives with respect to color. If the symbol is not of the target's color, no further examination is required. If it is of the target's color, then a further search is made that involves $\log _{2} 60$ bits. Thus, the total information is $30+15 \log _{2} 60$ bits.

Second, there are 45 symbols presented of three colors, each of 15 symbols. To identify the color of 1 symbol requires $\log _{2} 3$ bits. Therefore, the total information with respect to color is $45 \log _{2} 3$ bits. If a symbol of the target's color is located, a further search for number, requiring $\log _{2} 60$ bits, is utilized. The total information is given by $45 \log _{2} 3+15$ $\log _{2} 60$ bits.

Finally, by similar analysis, the

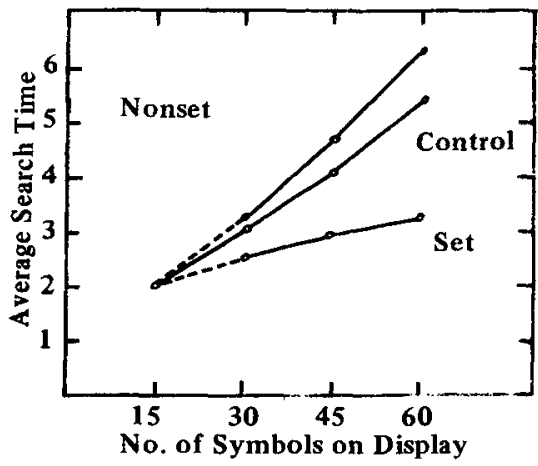

Fig. 3. Average search time as a function of number of symbols on display for Green and Anderson's second experiment (reproduced from Green \& Anderson, 1956).

four-color situation gives a total information count of $60 \log _{2} 4+15 \log _{2} 60$ bits.

These information values, together with those from the control group, are plotted in Fig. 4.

As in Experiment 1, the data points from both conditions - set and control-lie on the same straight line, with an information-transmission rate of 80 $\mathrm{bits} / \mathrm{sec}$ (again, on average, $40 \mathrm{bits} / \mathrm{sec}$ ). Although two different groups of Ss were used in the two experiments, the slopes and intercepts of both of the straight lines in Figs. 2 and 4 can be described by the same parameters. Thus, we can represent Experiments 1 and 2 on the same graph by equating them in terms of their information content. This graph is shown in Fig. 5.

It is clear from Fig. 5 that an informational analysis of these two separate experiments provides an adequate description of the tasks involved.

\section{DISCUSSION}

As we remarked earlier in this paper, the nonset group in Experiment 1 should have a constant search time. The Ss in this situation would appear to be searching for 1 of 60 numbers, the number of symbols of one or the other color being irrelevant to their search. In our analysis, this would be equivalent to a search involving 60 $\log _{2} 60$ bits.

One explanation of the inverted- $U$ relation found by Green and Anderson is that the $S$ s do not use a systematic line-by-line search but, rather, employ a search strategy that is, in many cases, highly redundant. Thus, in this case, there are two colors, red and green, that are randomly distributed among the array; if we assume that the $S$ scans first one color and then the other (the $S$ not knowing the 


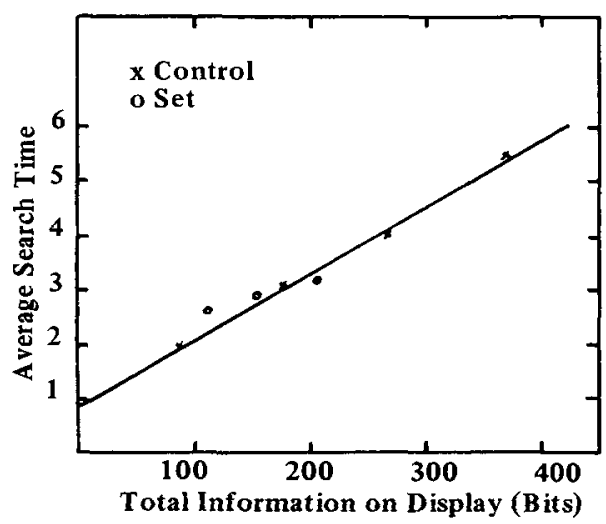

Fig. 4. Average search time as a function of total information on display.

target color), then, for randomly distributed numbers, the most wasteful amount of scanning-due to repeated overlap of previously scanned areas-will occur when the two colors are present in equal ratios, i.e., 30:30. Similarly, minimum time wastage will occur for the two extreme cases when there are only a minimal number of one of the colors present (i.e., 10:50 and 50:10).

Such an approach would lead to an inverted- $U$ relation between search time and the proportion of target color in the array. Any subsequent informational analysis of this particular case would first have to be corrected for the redundant strategy (redundant in the sense of overlap and duplication of areas scanned if first one color is checked and then the other, rather than a line-by-line scan).

A similar argument may be advanced for the nonset group in Experiment 2. If the Ss use a redundant strategy (as defined above), then the more colors there are in a given display, the more overlap and duplication of symbols that will occur. Thus, at a qualitative level, one would predict that the discrepancy between the search times for the nonset and control conditions increase progressively as the number of colors in equivalent displays (in terms of total number of symbols) increases. Examination of Fig. 3 shows this to be the case.

It would seem from the analysis of the other conditions that optimum search strategies have been used, and that the subsequent informational analysis has fulfilled the initial predictions. Namely, that when the various informational weightings have been given to each separate condition, all the data may be accounted for by one information-theory model, and the constant transmission rate shown by the Ss yield a linear relation between search time and amount of information on display. Further predictions concerning expected search times in other visual-search tasks may then be made as a consequence of this model, once the appropriate transmission rate has been determined.

With respect to the value of the transmission rate obtained in this analysis, it should be noted that since the total possible array consisted of 60 symbols (two digits, 10-69), $\mathrm{n}$, the size of the array, was taken to be equal to 60 for all subsequent calculations (i.e., control condition: number of symbols, $x$, then total information in the display equals $x \log 60$ ). This is not necessarily so. The $S$ may have "defined" his array size as something different from this. Thus, he might search from an array covering the complete range for two-digit numbers, i.e., $00-99$, and then $n$ would equal 100 , and the final calculated transmission rate would be increased somewhat. This would serve on the present model to increase the transmission rate. However, since a good linear plot was obtained, implying a constant value for $n$, all Ss used either a value of 60 or a value of 100 (say). The former is the most likely, since the Ss were allowed several practice trials that would presumably familiarize them with the range of the two-digit values.

Another point of reference is that, in this analysis, we have assumed a systematic search for color followed, in the appropriate cases, by a search for number-a two-digit number. In fact, an alternative procedure for the number search is to first consider the color, as before, but then to consider only the first digit of the two-digit number and, finally, the second digit if, and only if, the first digit conforms to the target requirements. Such a search strategy would theoretically be more economical than treating the two-digit number as one "unit." However, an analysis conforming to this strategy does not yield as good a fit as the "two-digit-unit" approach. We therefore conclude that the Ss do not treat the digits separately.

The authors are at present investigating

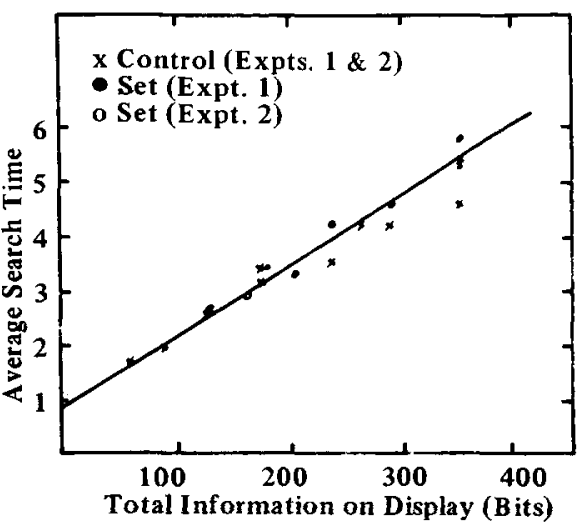

Fig. 5. Average search time as a function of total information on display (combined data from Figs. 2 and 4).

the information characteristics under free search and forced line-by-line strategies for numbers made up of increasing numbers of digits, in order to ascertain "unit" size, and for similar arrays, e.g., letters of the alphabet. The effect of false instructions to Ss concerning the possible size of the array appears to offer a potentially rewarding field of study with respect to the Ss' decision-making processes and comparitor mechanisms.

In conclusion, we would like to point out that strategy of search affords an important step in realizing macro-mechanisms of the human brain, and that information-theory approaches have the dual advantage of quantifying the steps, or units, as well as reflecting the mechanism or path. Even in those cases where search strategy exhibits varying degrees of redundancy, the value of the redundancy, so calculated, may aid in ascertaining search or decision mechanisms.

\section{REFERENCES}

COHEN, J., \& MEUDELL, P. Strategies of search. Acta Psychologica, 1968, 28, 322-343

COWAN, T. M. An observing response analysis of visual search. Psychological Review, 1968, 75, 265-270.

GREEN, B. F., \& ANDERSON, L. K. Color coding in a visual search task. Journal of Experimental Psychology, 1956, 51, 19-24.

\section{NOTE}

1. Address: Department of Psychology, The University of Manchester, Manchester, England.

(Accepted for publication June 6, 1969.) 